\title{
Noether Symmetries and Conserved Quantities of $f(R)$ Cosmology Models Containing Time Transformation ${ }^{1}$
}

\author{
Jing-Li $\mathrm{Fu}^{1^{*}}$ and Yong-Xin Guo ${ }^{2}$ \\ ${ }^{1}$ Institute of Mathematical Physics, Zhejiang Sci-Tech University, Hangzhou 310018, China \\ ${ }^{2}$ Department of Physics, Liaoning University, Shenyang 110036,China \\ Email: sqfujingli@163.com
}

\begin{abstract}
According to the invariance of Hamilton action of the generic $f(R)$ cosmological model under the infinitesimal transformations with respect to the time $t$, the scale factor $a$ and the Ricci scalar $R$, the generalized variation principle of the $f(R)$ cosmological model is presented. The definition and the criterion of Noether symmetry are given for this model. The Noether identical equation and the Noether theorem of generic $f(R)$ cosmological models are obtained. The Lie group of transformation, the conserved quantity and the form of generic $f(R)$ gravity are derived via the method of Noether symmetry. It is also shown that the resulting form of $f(R)$ yields as conservative law of the cosmological scale factor. Finally, an example is discussed to illustrate these results.
\end{abstract}

Keywords: $f(R)$ cosmological model, Noether symmetry, conserved quantity, time transformation.

\section{Introduction}

In recent years, modified theories of gravity have been constructed by adding correction terms to the Einstein-Hilbert action, which have opened a new window to study the accelerated expansion of the universe. It has shown that such correction terms could give rise to accelerating solutions of the field equations without having to invoke concepts such as dark energy [1]. In a more general setting, one can use a generic function $f(R)$, instead of the usual Ricci scalar $R$ as the action of general relativity. Such $f(R)$ gravity theories have been extensively explained in literatures over the past few years [2]. Now, Nojiri1 and Odintsov have presented the versions of $f(R), f(G)$ or $f(R, G)$ gravity models with non-linear gravitational coupling or string-inspired model with auss-Bonnet-dilaton coupling in the late universe where they lead to cosmic speed-up [3,4]. In finding the dynamical equations of motion one can vary the action with respect to the metric (metric formalism), or view the metric and connections as independent dynamical variables and vary the action with respect to both independently (Palatini formalism) [5]. In this theory, the Palatini form of the action is shown to be equivalent to a scalar-tensor type theory from which the scalar field kinetic energy is absent. This is achieved by introducing a conformal transformation in which the conformal factor is taken as an auxiliary scalar field [6]. As is well known, in the usual Einstein-Hilbert action these two approaches give the same field equations. However, in $f(R)$ gravity the Palatini formalism leads to different dynamical equations due to nonlinear terms in the action. There is also a third version of $f(R)$ gravity in which the Lagrangian of the matter depends on the connections of the metric (metric-affine formalism) [7].

On the other hand, Lie group theory, which can be used to solve differential equations, to study constrained mechanical systems, to investigate mechanico-electrical systems and to establish properties of their solution space, has been proved to be a powerful tool. These aspects of Lie group theory have been described in many literatures [8 15]. The symmetry of mechanical systems is one of the most important subjects in physics, which has been investigated for a long time. The symmetry of a mechanical system will be useful for integrating the equations of motion, since it is closely related to invariants (or first integrals) of a system. The modern approaches of finding invariants are mainly in terms of the Noether symmetry and the Lie symmetry. The Noether symmetry is an invariance of the action integral under the infinitesimal transformation of a continuous group. The Noether's theorem

\footnotetext{
1 Project supported by the National Natural Science Foundation of China (Grant No. 11272287,11472247)
} 
associates one conserved quantity to the Noether symmetry. Lie's method consists in finding the continuous symmetry transformations that leaves a system of differential equations invariant. These symmetry transformations constitute the Lie group.

Recently, the symmetry theories have been applied to cosmological models. Camci and Kucukakca presented the Noether symmetry of Bianchi I, Bianchi III, and Kantowski-Sachs cosmological models, and obtained solutions of the field equations for the considered models using the results obtained from the Noether symmetry [16]. Sanyal, Modak, Rubano and Piedipalumbo studied the Noether symmetry in the higher order gravity theory, and gave the correct quantum description of the theory [17]. Capozziello, Stabile and Troisi searched for spherically symmetric solutions of $f(R)$ theories of gravity via the Noether symmetry approach [18]. Vakili investigated the Noether symmetries of generic $f(R)$ cosmological model, and further using approach of Noether symmetry quantized a flat FRW cosmology of the $f(R)$ gravity $[19,20]$. However, in foregoing works, the Noether symmetries of the $f(R)$ cosmology models are only the invariance of Lagrangian under the infinitesimal transformation for the scale factor $a$ and the scalar $R$. In this paper, great efforts are made on expanding the theory of Noether symmetries of $f(R)$ cosmology models to a more generalized form which includes the infinitesimal transformations of the time $t$, the scale factor $a$ and the scalar $R$. Works on the Noether symmetry of $f(R) \operatorname{cosmology}$ models which may be regarded as special cases of this paper will be presented in a parallel paper to illustrate a generalized approach of Noether symmetry for explaining $f(R)$ cosmology models. The approach of Lie group analysis is extremely attractive in applications for generic $f(R)$ cosmology models, because this method can determine the forms of $f(R)$ and scale factor a for $f(R)$ cosmology models and derive the conserved quantities (including energy conservative, momentum conservative, angle momentum conservative or other forms of conservative) corresponding to $f(R)$ cosmology models. This method can also provide the solution of $f(R)$ cosmology models.

In this paper, the infinitesimal transformations of $f(R)$ cosmological models with respect to the time $t$, the scale factor $a$ and the Ricci scalar $R$ are introduced. Secondly, we consider the variation of Hamiltonian action associated with Lagrangian to present the Hamilton variation principle of $f(R)$ cosmological models. Further, we derive the Noether identity (i.e. the Lie derivative of the Lagrangian with respect to this vector field vanishes) and Noether conserved quantity. The Noether symmetry of Lagrangian containing time, the scale factor $a$ and the Ricci scalar $R$ is discussed and the explicit form of the function $f(R)$ is obtained. Since the existence of symmetry results in a constant of motion, one can integrate the field equations which would then lead to a power law expansion of the universe. We also present the theory of Noether symmetry for Palatini $f(R)$ cosmology model with an example.

\section{Hamiltonian Action of $f(R)$ Cosmology Model}

In this section, we consider a spatially flat FRW cosmology within the framework of $f(R)$ gravity. The $(n+1)$-dimensional action (we work in units where $c=16 \pi \mathbf{G}=1$ )

$$
s=\int d^{n+1} x \sqrt{-g} f(R) .
$$

where $R$ is the scalar curvature and $f(R)$ is an arbitrary function of $R$. By varying the above action with respect to metric, the equation of motion is obtained as [19]

$$
\frac{1}{2} g_{\mu \nu} f(R)-R_{\mu \nu} f^{\prime}(R)+\nabla_{\mu} \nabla_{v} f^{\prime}(R)-g_{\mu \nu} \square f^{\prime}(R)=0,
$$

where a prime represents differentiation with respect to $R$. It is assumed that the geometry of spacetime is described by the flat FRW metric which seems to be consistent with the present cosmological observations.

$$
\mathrm{d} s^{2}=-\mathrm{d} t^{2}+a^{2}(t) \sum_{i=1}^{n}\left(\mathrm{~d} x^{i}\right)^{2} .
$$

With this background geometry the field equations read

$$
(n-1) \dot{P}+\frac{n(n-1)}{2} P^{2}=-\frac{1}{f^{\prime}}\left[f^{\prime \prime \prime} \dot{R}^{2}+(n-1) P \dot{R} f^{\prime \prime}+f^{\prime \prime} \ddot{R}+\frac{1}{2}\left(f-R f^{\prime}\right)\right],
$$




$$
P^{2}=\frac{1}{n(n-1) f^{\prime}}\left[\left(f^{\prime} R-f\right)-2 n \dot{R} P f^{\prime \prime}\right]
$$

where $P=\dot{a} / a$ is the Hubble parameter and a dot represents differentiation with respect to $t$. To study the symmetries of the minisuperspace under consideration, an effective Lagrangian is needed for the model whose variation with respect to its dynamical variables yields the correct equations of motion. The action with the scale factor $a$ and scale curvature $R$ can be written in the form [21]

$$
S=\int \mathrm{d} t L(a, \dot{a}, R, \dot{R})=\int \mathrm{d} t\left\{a^{n} f(R)-\lambda\left[R-n(n-1) \frac{\dot{a}^{2}}{a^{2}}-2 n \frac{\ddot{a}}{a}\right]\right\} .
$$

where we introduce the definition of $R$ in terms of $a$ and its derivative is a constant. This procedure allows us to remove the second order derivatives from action (6). The Lagrange multiplier $\lambda$ can be obtained by variation with respect to $R$, that is, $\lambda=a^{n} f^{\prime}(R)$. Thus, the Lagrangian for the $f(R) \operatorname{cosmology}$ model can be expressed by

$$
L(a, \dot{a}, R, \dot{R})=n(n-1) \dot{a}^{2} a^{n-2} f^{\prime}+2 n \dot{a} \dot{R} a^{n-1} f^{\prime \prime}+a^{n}\left(f^{\prime} R-f\right) .
$$

The Hamilton action of the $f(R)$ cosmology model (6) is then expressed in the form

$$
S(\gamma)=\int_{t_{0}}^{t} \mathrm{~d} t L(a, \dot{a}, R, \dot{R})=\int_{t_{0}}^{t}\left[n(n-1) \dot{a}^{2} a^{n-2} f^{\prime}+2 n \dot{a} \dot{R} a^{n-1} f^{\prime \prime}+a^{n}\left(f^{\prime} R-f\right)\right] \mathrm{d} t,
$$

where $\gamma$ is an arbitrary curve.

\section{Hamilton Variation Principle for $f(R)$ Cosmology Model}

Introduce infinitesimal transformations of the time $t$, the scale factor $a$ and the scale curvature $R$

$$
t^{*}=t+\Delta t, \quad a^{*}=a+\Delta a, \quad R^{*}=R+\Delta R,
$$

where isochronous variations are used. Their expanded forms are

$$
t^{*}=t+\varepsilon \xi_{0}(t, a, R), \quad a^{*}=a+\varepsilon \xi(t, a, R), \quad R^{*}=R+\varepsilon \eta(t, a, R),
$$

where $\varepsilon$ is a small parameter, and $\xi_{0}, \xi$ and $\eta$ are the infinitesimal. Hamiltonian action $S(\gamma)$ associated with $f(R)$ cosmology model under the infinitesimal transformation (10) is written as

$$
S\left(\gamma^{*}\right)=\int_{t_{0}}^{t} L\left(a^{*}, \dot{a}^{*}, R^{*}, \dot{R}^{*}\right) \mathrm{d} t^{*}
$$

where $\mathrm{d} t^{*}$ is written as

$$
\mathrm{d} t^{*}=\mathrm{d} t+\varepsilon \mathrm{d}(\Delta t)=\left[1+\frac{\mathrm{d}(\Delta t)}{\mathrm{d} t}\right] \mathrm{d} t .
$$

Application of the variation calculus to the Hamiltonian action $S(\gamma)$ leads to

$$
\Delta S=S\left(\gamma^{*}\right)-S(\gamma) \approx \int_{t_{0}}^{t}\left[L \frac{d(\Delta t)}{d t}+\frac{\partial L}{\partial t} \Delta t+\frac{\partial L}{\partial a} \Delta a+\frac{\partial L}{\partial R} \Delta R+\frac{\partial L}{\partial \dot{a}} \Delta \dot{a}+\frac{\partial L}{\partial \dot{R}} \Delta \dot{R}\right] \mathrm{d} t,
$$

where only the linear term of small parameter $\varepsilon$ is taken into consideration. That is, all non-linear terms of small parameter $\varepsilon$ are neglected in the variation $\Delta S$.

The relationships between the isochronous variation and the complete variation are given by

$$
\begin{aligned}
& \Delta a=\delta a+\dot{a} \Delta t, \quad \Delta R=\delta R+\dot{R} \Delta t, \quad \Delta \dot{a}=\delta \dot{a}+\ddot{a} \Delta t, \quad \Delta \dot{R}=\delta \dot{R}+\ddot{R} \Delta t, \\
& \delta \dot{a}=\frac{\mathrm{d}}{\mathrm{d} t} \delta a, \quad \delta \dot{R}=\frac{\mathrm{d}}{\mathrm{d} t} \delta R .
\end{aligned}
$$

Substituting Eq. (14) into (13) yields

$$
\left.\Delta S=\int_{t_{1}}^{t_{2}}\left\{\frac{\mathrm{d}}{\mathrm{d} t} L \Delta t+\frac{\partial L}{\partial \dot{a}} \delta a+\frac{\partial L}{\partial \dot{R}} \delta R\right)+\left(\frac{\partial L}{\partial a}-\frac{\mathrm{d}}{\mathrm{d} t} \frac{\partial L}{\partial \dot{a}}\right) \delta a+\left(\frac{\partial L}{\partial R}-\frac{\mathrm{d}}{\mathrm{d} t} \frac{\partial L}{\partial \dot{R}}\right) \delta R\right\} \mathrm{d} t .
$$

Equation (15) is a fundamental variation formula of the Hamiltonian action of $f(R)$ cosmology models. Inserting Eq. (10) into (15) leads to

where

$$
\Delta S=\int_{t_{0}}^{t}\left\{\frac{\mathrm{d}}{\mathrm{d} t}\left(L \xi_{0}+\frac{\partial L}{\partial \dot{a}} \bar{\xi}+\frac{\partial L}{\partial \dot{R}} \bar{\eta}\right)+\left(\frac{\partial L}{\partial a}-\frac{\mathrm{d}}{\mathrm{d} t} \frac{\partial L}{\partial \dot{a}}\right) \bar{\xi}+\left(\frac{\partial L}{\partial R}-\frac{\mathrm{d}}{\mathrm{d} t} \frac{\partial L}{\partial \dot{R}}\right) \bar{\eta}\right\} \mathrm{d} t,
$$




$$
\bar{\xi}=\xi-\dot{a} \xi_{0}, \quad \bar{\eta}=\eta-\dot{R} \xi_{0}
$$

\section{Noether Theory of the $f(R)$ Cosmology Model}

It is well known that a system has the Noether symmetry when its Hamilton action is invariant under an infinitesimal transformation with respect to time-space variable. The definitions and the criterions of Noether symmetry transformations for $f(R)$ cosmology models are given as follow:

Definition 1. If the Hamiltonian action (8) of an $f(R)$ cosmology model is invariant under an infinitesimal transformation (10) with respect to the time $t$, the scale factor $a$ and the Ricci scalar $R$, and the following condition

$$
\Delta S=0,
$$

always holds, then the transformation is called the Noether symmetry transformation associated with the model.

Combining definition 1 with Eqs. (15) and (16) leads to the following criterions:

Criterion 1. Infinitesimal transformation (9) is a Noether symmetry transformation of $f(R)$ cosmology model, on the condition that

$$
\frac{\partial L}{\partial t} \Delta t+\frac{\partial L}{\partial a} \Delta a+\frac{\partial L}{\partial R} \Delta R+\frac{\partial L}{\partial \dot{a}} \Delta \dot{a}+\frac{\partial L}{\partial \dot{R}} \Delta \dot{R}+L \frac{\mathrm{d}}{\mathrm{d} t}(\Delta t)=0
$$

Criterion 2. Infinitesimal transformation (9) is a Noether symmetry transformation of an $f(R)$ cosmology model, if equation

$$
\frac{\mathrm{d}}{\mathrm{d} t}\left(L \xi_{0}+\frac{\partial L}{\partial \dot{a}} \bar{\xi}+\frac{\partial L}{\partial \dot{R}} \bar{\eta}\right)+\left(\frac{\partial L}{\partial a}-\frac{\mathrm{d}}{\mathrm{d} t} \frac{\partial L}{\partial \dot{a}}\right) \bar{\xi}+\left(\frac{\partial L}{\partial R}-\frac{\mathrm{d}}{\mathrm{d} t} \frac{\partial L}{\partial \dot{R}}\right) \bar{\eta}=0
$$

holds.

Consider the following relationships

$$
\Delta t=\varepsilon \xi_{0}(t, a, R), \quad \Delta a=\varepsilon \xi(t, a, R), \quad \Delta R=\varepsilon \eta(t, a, R),
$$

then Eq. (19) is written as

$$
\frac{\partial L}{\partial t} \xi_{0}+\frac{\partial L}{\partial a} \xi+\frac{\partial L}{\partial R} \eta+\frac{\partial L}{\partial \dot{a}}\left(\dot{\xi}-\dot{a} \dot{\xi}_{0}\right)+\frac{\partial L}{\partial \dot{R}}\left(\dot{\eta}-\dot{R}^{\dot{\xi}_{0}}\right)+L \dot{\xi}_{0}=0
$$

we call the Eq.(22) a Noether identity associated with the $f(R)$ cosmology model with infinitesimal transformation (10).

The Noether symmetry transformation of the $f(R)$ cosmology model can be determined by Criterion 1 and Criterion 2.

The Noether theorem is given as follows:

Theorem 1. If the infinitesimal transformation (10) is Noether symmetry transformation, the $f(R)$ cosmology model possesses linear independent first integral

$$
I=L \xi_{0}+\frac{\partial L}{\partial \dot{a}} \bar{\xi}+\frac{\partial L}{\partial \dot{R}} \bar{\eta}=\text { const. }
$$

Proof. The Noether symmetry transformation (10) makes Eqs. (16) and (18) hold. Eq. (16) and (18) can be expressed as

$$
\Delta S=\int_{t_{0}}^{t}\left\{\frac{\mathrm{d}}{\mathrm{d} t}\left(L \xi_{0}+\frac{\partial L}{\partial \dot{a}} \bar{\xi}+\frac{\partial L}{\partial \dot{R}} \bar{\eta}\right)+\left(\frac{\partial L}{\partial a}-\frac{\mathrm{d}}{\mathrm{d} t} \frac{\partial L}{\partial \dot{a}}\right) \bar{\xi}+\left(\frac{\partial L}{\partial R}-\frac{\mathrm{d}}{\mathrm{d} t} \frac{\partial L}{\partial \dot{R}}\right) \bar{\eta}\right\} \mathrm{d} t
$$

Considering arbitrariness of the integral region $\left[t_{1}, t_{2}\right]$ and using Lagrange equations of the $f(R)$ cosmology model, one obtains

$$
\frac{\mathrm{d}}{\mathrm{d} t}\left(L \xi_{0}+\frac{\partial L}{\partial \dot{a}} \bar{\xi}+\frac{\partial L}{\partial \dot{R}} \bar{\eta}\right)=0
$$

Theorem 2. If the infinitesimal transformation (10) satisfies Noether identity $(22)$, the $f(R)$ cosmology model possesses linear independent first integral (23). 


\section{Noether Symmetry Solution of $f(R)$ Cosmology Model}

It is well known that the Noether symmetry approach is a powerful tool in finding the solution to a given Lagrangian, including the one presented above. Using this approach, one can obtain conserved quantities and solutions associated with the systems. Here, the aim is to find the function $f(R)$ such that the corresponding $f(R)$ cosmology model exhibits the desired symmetry.

By substituting Lagrangian (7) into Noether identity (22), one has

$$
\begin{aligned}
& n(n-1)(n-2) \dot{a}^{2} a^{n-3} f^{\prime} \xi+2 n(n-1) \dot{a} \dot{R} a^{n-2} f^{\prime \prime} \xi+n a^{n-1}\left(f^{\prime} R-f\right) \xi \\
& +n(n-1) \dot{a}^{2} a^{n-2} f^{\prime \prime} \eta+2 n \dot{a} \dot{R} a^{n-1} f^{\prime \prime \prime} \eta+a^{n} f^{\prime \prime} R \eta+ \\
& +2 n(n-1) \dot{a} a^{n-2} f^{\prime}\left(\frac{\partial \xi}{\partial t}+\dot{a} \frac{\partial \xi}{\partial a}+\dot{R} \frac{\partial \xi}{\partial R}-\dot{a} \frac{\partial \xi_{0}}{\partial t}-\dot{a}^{2} \frac{\partial \xi_{0}}{\partial a}-\dot{a} \dot{R} \frac{\partial \xi_{0}}{\partial R}\right) \\
& +2 n \dot{R} a^{n-1} f^{\prime \prime}\left(\frac{\partial \xi}{\partial t}+\dot{a} \frac{\partial \xi}{\partial a}+\dot{R} \frac{\partial \xi}{\partial R}-\dot{a} \frac{\partial \xi_{0}}{\partial t}-\dot{a}^{2} \frac{\partial \xi_{0}}{\partial a}-\dot{a} \dot{R} \frac{\partial \xi_{0}}{\partial R}\right)+ \\
& +2 n \dot{a} a^{n-1} f^{\prime \prime}\left(\frac{\partial \eta}{\partial t}+\dot{a} \frac{\partial \eta}{\partial a}+\dot{R} \frac{\partial \eta}{\partial R}-\dot{R} \frac{\partial \xi_{0}}{\partial t}-\dot{a} \dot{R} \frac{\partial \xi_{0}}{\partial a}-\dot{R}^{2} \frac{\partial \xi_{0}}{\partial R}\right) \\
& +n(n-1) a^{n-2} f^{\prime} \frac{\partial \xi_{0}}{\partial t} \dot{a}^{2}+2 n a^{n-1} f^{\prime \prime} \frac{\partial \xi_{0}}{\partial t} \dot{a} \dot{R}+a^{n}\left(f^{\prime} R-f\right) \frac{\partial \xi_{0}}{\partial t} \\
& +n(n-1) a^{n-2} f^{\prime} \frac{\partial \xi_{0}}{\partial a} \dot{a}^{3}+2 n a^{n-1} f^{\prime \prime} \frac{\partial \xi_{0}}{\partial a} \dot{a}^{2} \dot{R}+a^{n}\left(f^{\prime} R-f\right) \frac{\partial \xi_{0}}{\partial a} \dot{a} \\
& +n(n-1) a^{n-2} f^{\prime} \frac{\partial \xi_{0}}{\partial R} \dot{a}^{2} \dot{R}+2 n a^{n-1} f^{\prime \prime} \frac{\partial \xi_{0}}{\partial R} \dot{a} \dot{R}^{2}+a^{n}\left(f^{\prime} R-f\right) \frac{\partial \xi_{0}}{\partial R} \dot{R}=0 .
\end{aligned}
$$

Thus, the resulting expression is identically equal to zero if and only if these coefficients of $\dot{a}, \dot{R}, \dot{a}^{2}, \cdots, \dot{a}^{3}, \cdots, \dot{a} \dot{R}^{2}$ are zero. This leads to a system of partial differential equations for $\xi_{0}$, $\xi$ and $\eta$. Condition (26) results in

$$
\begin{gathered}
n\left(f^{\prime} R-f\right) \xi+a f^{\prime \prime} R \eta+a\left(f^{\prime} R-f\right) \frac{\partial \xi_{0}}{\partial t}=0 \\
2 n(n-1) f^{\prime} \frac{\partial \xi}{\partial t}+2 n a f^{\prime \prime} \frac{\partial \eta}{\partial t}+a^{2}\left(f^{\prime} R-f\right) \frac{\partial \xi_{0}}{\partial t}=0 \\
2 n f^{\prime \prime} \frac{\partial \xi}{\partial t}+a\left(f^{\prime} R-f\right) \frac{\partial \xi_{0}}{\partial R}=0 \\
(n-1)(n-2) f^{\prime} \xi+(n-1) a f^{\prime \prime} \eta+2(n-1) a f^{\prime} \frac{\partial \xi}{\partial a}-(n-1) a f^{\prime} \frac{\partial \xi_{0}}{\partial t}+2 a^{2} f^{\prime \prime} \frac{\partial \eta}{\partial a}=0, \\
f^{\prime \prime} \frac{\partial \xi}{\partial R}=0 \\
(n-1) f^{\prime \prime} \xi+a f^{\prime \prime \prime} \eta+a f^{\prime \prime} \frac{\partial \eta}{\partial R}+(n-1) f^{\prime} \frac{\partial \xi}{\partial R}-a f^{\prime \prime} \frac{\partial \xi_{0}}{\partial t}+a f^{\prime \prime} \frac{\partial \xi}{\partial a}=0, \\
f^{\prime} \frac{\partial \xi_{0}}{\partial a}=0, \\
-(n-1) f^{\prime} \frac{\partial \xi_{0}}{\partial R}-2 a f^{\prime \prime} \frac{\partial \xi_{0}}{\partial a}-2 a f^{\prime \prime} \frac{\partial \xi_{0}}{\partial R}=0 .
\end{gathered}
$$

When we, from Eq.(33), take

$$
\xi_{0}=c_{0}=\text { const. }
$$

Equations (27) (34) have the following forms

$$
\begin{gathered}
n\left(f^{\prime} R-f\right) \xi+a f^{\prime \prime} R \eta=0, \\
2 n(n-1) f^{\prime} \frac{\partial \xi}{\partial t}+2 n a f^{\prime \prime} \frac{\partial \eta}{\partial t}=0,
\end{gathered}
$$




$$
\begin{gathered}
2 n f^{\prime \prime} \frac{\partial \xi}{\partial t}=0, \\
(n-1)(n-2) f^{\prime} \xi+(n-1) a f^{\prime \prime} \eta+2(n-1) a f^{\prime} \frac{\partial \xi}{\partial a}+2 a^{2} f^{\prime \prime} \frac{\partial \eta}{\partial a}=0, \\
f^{\prime \prime} \frac{\partial \xi}{\partial R}=0, \\
(n-1) f^{\prime \prime} \xi+a f^{\prime \prime \prime} \eta+a f^{\prime \prime} \frac{\partial \eta}{\partial R}+(n-1) f^{\prime} \frac{\partial \xi}{\partial R}+a f^{\prime \prime} \frac{\partial \xi}{\partial a}=0 .
\end{gathered}
$$

Equation (31) gives $f^{\prime \prime}=0$ or $\partial \xi / \partial R=0$. In the case where $f^{\prime \prime}=0$, we obtain

$$
f(R)=c_{1} R+c_{2},
$$

Substituting this result into Eq.(36) we get $c_{2}=0$, and recover the usual Einstein-Hilbert gravity without cosmological constant for which $f(R)=R$. Also, since Eq.(41) results in $\partial \xi / \partial R=0$, from Eq.(39) we obtain the following differential equation on $\xi(a)$

$$
(n-2) \xi(a, t)+2 a \frac{\partial \xi}{\partial a}=0 .
$$

On the other hand, substituting Eq.(42) into (37), one has

then Eqs.(43) and (44) have solution

$$
\frac{\partial \xi}{\partial t}=0, \quad \text { i.e, } \quad \xi=\xi(a)
$$

$$
\xi(a)=a^{-\frac{n-2}{2}} .
$$

When $\dot{f}^{\prime}=0$, the Lagrangian (7) is independent on $R$, therefore, for arbitrary function $\eta(t, a, R)$, using Eqs.(23) and (45), the system (7) possesses the Noether conserved quantity:

$$
I=-n(n-1) \dot{a}^{2} a^{n-2} c_{0}+2 n(n-1) \dot{a} a^{n-2} a^{-\frac{n-2}{2}}=\text { const. }
$$

By taking $c_{0}=0$, the system possesses the Noether conserved quantity as

$$
I_{0}=a^{-\frac{n-2}{2}} \dot{a} a^{n-2}=\text { const. }
$$

which results in

$$
a(t) \square t^{\frac{n}{2}}
$$

Although, this solution for the scale factor satisfies the field equation (4), it does not satisfy the Hamiltonian constraint (5). This is not surprising since it is well known that the flat FRW cosmology with Einstein-Hilbert action (without the cosmological constant) has no vacuum solution. Thus, we remove the case $f^{\prime \prime}=0$ from our consideration. When $f^{\prime \prime} \neq 0$, from Eqs. (40) and (38) we obtain

$$
\frac{\partial \xi}{\partial R}=0
$$

and

$$
\frac{\partial \xi}{\partial t}=0
$$

Substituting Eq.(50) into (37) leads to

$$
\frac{\partial \eta}{\partial t}=0, \text { i.e } \cdot \eta=\eta(a, R)
$$

Using conditions (49) (51), the Eq.(36) results in

$$
\xi(a)=\frac{a f^{\prime \prime} R}{n\left(f-f^{\prime} R\right)} \eta(a, R) .
$$

When we substitute this result into Eq.(39) to eliminate $\xi(a)$, one yields

$$
\frac{\partial \eta}{\partial a}=\frac{n(n-1) f}{2 a\left(f^{\prime} R-n f\right)} \eta(a, R) .
$$


This equation can be solved by the approach of apart variable. We let

Substituting this equation into (53) we obtain

$$
\eta(a, R)=x(a) y(R)
$$

$$
\frac{2 a}{x} \frac{\mathrm{d} x}{\mathrm{~d} a}=\frac{n(n-1) f}{f^{\prime} R-n f}
$$

Since the left-hand side of this equation is a function of $a$ only while the right-hand side is a function of $R$, one should have

$$
\frac{n(n-1) f}{f^{\prime} R-n f}=c,
$$

and

$$
\frac{2 a}{x} \frac{\mathrm{d} x}{\mathrm{~d} a}=c
$$

Solve Eq.(56), one has

$$
f(R)=R^{\frac{n(n+c-1)}{c}},
$$

where the constant $c \neq 0$. is required to have $f^{\prime \prime} \neq 0$.

The Eq.(57) results in

$$
x(a)=a^{\frac{c}{2}} .
$$

Now, using Eqs.(54), (58) and (59) in Eq.(52), we obtain

$$
\xi(a)=-\frac{n+c-1}{c} R^{-1} a^{\frac{c}{2}+1} B(R) .
$$

Since $\xi(a)$ should be a function of $a$ only, from the Eq.(60) we conclude that $y(R)=R$ and thus obtain Lie group of transformation as

$$
\xi_{0}=c_{0}, \eta(a, R)=R a^{c / 2}, \xi(a)=-\frac{n+c-1}{c} a^{\frac{c}{2}+1} .
$$

To determine the constant $c$, by substituting results (52), (58) and (61) into (39) we find

$$
c=-(n+1) \text {. }
$$

Now, the Noether symmetry, which satisfies Noether identical equation (22), associated with the Hamilton action of the $f(R)$ cosmology $(6)$ is given by

$$
\xi_{0}=c_{0}, \quad \xi(a)=-\frac{2}{n+1} a^{-\frac{n-1}{2}}, \quad \eta(a, R)=R a^{-\frac{n+1}{2}},
$$

and

$$
f(R)=R^{\frac{2 n}{n+1}}
$$

According to Noether theorem 2, substituting Eqs.(63) and (64) into (23), the conserved quantity corresponding to $f(R)$ cosmology model is obtained by

$$
\begin{aligned}
& I=L \xi_{0}+\frac{\partial L}{\partial \dot{a}} \bar{\xi}+\frac{\partial L}{\partial \dot{R}} \bar{\eta} \\
& =\left[\frac{-2 n^{2}(n-1)}{(n+1)^{2}} \dot{a}^{2} a^{n-2} R^{\frac{n-1}{n+1}}-\frac{4 n^{2} n-1}{(n+1)^{3}} \dot{a} \dot{R} a^{n-1} R^{\frac{-2}{n+1}}+\frac{n-1}{n+1} a^{n} R^{\frac{2 n}{n+1}}\right] c_{0}(n+1) \\
& -\left[\frac{4 n^{2}(n-1)}{(n+1)^{2}} \dot{a} a^{\frac{n-3}{2}} R^{\frac{n-1}{n+1}}+\frac{8 n^{2}(n-1)}{(n+1)^{3}} \dot{R} a^{\frac{n-1}{2}} R^{-\frac{2}{n+1}}\right] .
\end{aligned}
$$

which can be written as

$$
I=\left[-\frac{4 n^{2}}{(n+1)^{2}} \frac{\mathrm{d}}{\mathrm{d} t}\left(a^{\frac{n-1}{2}} R^{\frac{n-1}{n+1}}\right)+\frac{n-1}{n+1} a^{n-2} R^{\frac{2 n}{n+1}}\right] c_{0}(n+1)-\frac{8 n^{2}}{(n+1)^{2}} \frac{\mathrm{d}}{\mathrm{d} t}\left(a^{\frac{n-1}{2}} R^{\frac{n-1}{n+1}}\right),
$$

If we take constant $c_{0}=1 / n+1$, then conserved quantity (66) can be written as 


$$
I=-\frac{6 n^{2}(n-1)}{(n+1)^{2}} \dot{a} a^{\frac{n-3}{2}} R^{\frac{n-1}{n+1}}-\frac{12 n^{2}(n-1)}{(n+1)^{3}} \dot{R} a^{\frac{n-1}{2}} R^{-\frac{2}{n+1}}+\frac{n-1}{n+1} a^{n} R^{\frac{2 n}{n+1}}=\text { const, }
$$

which may be rewritten in the form

$$
I=-\frac{12 n^{2}}{(n+1)^{2}} \frac{\mathrm{d}}{\mathrm{d} t}\left(a^{\frac{n-1}{2}} R^{\frac{n-1}{n+1}}\right)+\frac{n-1}{n+1} a^{n} R^{\frac{2 n}{n+1}}=\text { const. }
$$

Taking $c_{0}=0$, we obtain the result of Eq.(66) in [17]

$$
I=-\frac{8 n^{2}}{(n+1)^{2}} \frac{\mathrm{d}}{\mathrm{d} t}\left(a^{\frac{n-1}{2}} R^{\frac{n-1}{n+1}}\right)=\text { const }
$$

which can be immediately integrated assuming $\left.a(t)\right|_{t=0}=0$

$$
a^{\frac{n-1}{2}} R^{\frac{n-1}{n+1}}=-\frac{(n+1)^{2}}{8 n^{2}} I t .
$$

On the other hand, when we introduce generalized momentums associated with variables $a$ and $R$ as

$$
\begin{aligned}
& p_{1}=\frac{\partial L}{\partial \dot{a}}=\frac{4 n^{2}}{n+1}(n-1) \dot{a} a^{n-2} R^{\frac{n-1}{n+1}}+\frac{4 n^{2}}{n+1} \frac{n-1}{n+1} \dot{R} a^{n-1} R^{\frac{-2}{n+1}}, \\
& p_{2}=\frac{\partial L}{\partial \dot{R}}=\frac{4 n^{2}}{n+1} \frac{n-1}{n+1} \dot{a} a^{n-1} R^{\frac{-2}{n+1}}
\end{aligned}
$$

from above equations, we obtain

$$
\begin{aligned}
& \dot{a}=\frac{(n+1)^{2}}{4 n^{2}(n-1)} p_{2} a^{-(n-1)} R^{\frac{2}{n+1}} . \\
& \dot{R}=\frac{(n+1)^{2}}{4 n^{2}(n-1)}\left(p_{1}-(n+1) p_{2} a^{-1} R\right) a^{-(n-1)} R^{\frac{2}{n+1}} .
\end{aligned}
$$

We define Hamiltonian corresponding to Lagrangian (7) as

$$
\begin{aligned}
& H=p_{1} \dot{a}+p_{2} \dot{R}-L \\
& =\frac{4 n^{2}}{n+1}(n-1) \dot{a}^{2} a^{n-2} R^{\frac{n-1}{n+1}}+\frac{4 n^{2}}{n+1} \frac{n-1}{n+1} \dot{a} \dot{R} a^{n-1} R^{\frac{-2}{n+1}}+\frac{4 n^{2}}{n+1} \frac{n-1}{n+1} \dot{a} \dot{R} a^{n-1} R^{\frac{-2}{n+1}} \\
& \quad \quad-\left[\frac{2 n^{2}(n-1)}{n+1} \dot{a}^{2} a^{n-2} R^{\frac{n-1}{n+1}}+\frac{4 n^{2}}{n+1} \frac{n-1}{n+1} \dot{a} \dot{R} a^{n-1} R^{\frac{-2}{n+1}}+\frac{n-1}{n+1} a^{n} R^{\frac{2 n}{n+1}}\right] \\
& =\frac{2 n^{2}(n-1)}{n+1} \dot{a}^{2} a^{n-2} R^{\frac{n-1}{n+1}}+\frac{4 n^{2}(n-1)}{(n+1)^{2}} \dot{a} \dot{R} a^{n-1} R^{\frac{-2}{n+1}}-\frac{n-1}{n+1} a^{n} R^{\frac{2 n}{n+1}},
\end{aligned}
$$

According to Hamilton constraint $H=0$, we have

$$
2 n^{2} \dot{a}^{2} R^{-1}+\frac{4 n^{2}}{n+1} \dot{a} \dot{R} a R^{-2}=a^{2} .
$$

To obtain scale factor $a(t)$ from the above relation, note that Eq.(70) leads to

$$
\dot{R}=\left[-\frac{(n+1)^{2}}{8 n^{2}} I_{1}\right]^{\frac{n+1}{n-1}}\left(\frac{n+1}{n-1} t^{\frac{2}{n-1}} a^{-\frac{n+1}{2}}-\frac{n+1}{2} t^{\frac{n+1}{n-1}} \dot{a} a^{-\frac{n+3}{2}}\right),
$$

substituting this result into Eq.(74), one has

from this equation, we obtain

$$
\frac{4 n^{2}}{n-1}\left[-\frac{(n+1)^{2}}{8 n^{2}} I_{1}\right]^{\frac{n-1}{n+1}} \dot{a} a^{\frac{n-1}{2}}=t^{\frac{2 n}{n-1}},
$$

$$
a(t) \sim t^{\frac{2(3 n-1)}{n^{2}-1}} .
$$

Now, we should point out that for the context of $f(R)=R^{2 n / n+1}$ gravity, the universe evolves with a power law expansion (note that for any $n>1$ the power of the scale factor is positive). It is remarkable from (48) that the condition under which the universe would accelerate is $\left[2(3 n-1) / n^{2}-1\right]>1$, that is, $n \leq 5$. This means that models with spatial dimension $n \leq 5$ obey an accelerated power law expansion, while for $n>5$ a decelerated expansion occurs. 


\section{An Example}

Let us begin by introducing the action of the Palatini $f(R)$ theories

$$
S=\frac{1}{2 k} \int \mathrm{d}^{4} x \sqrt{-g} f(\bar{R})+S_{m}\left(g_{\mu \nu}, \psi_{m}\right)
$$

here $f(\tilde{R})$ is a function of $\tilde{R}=g_{\mu \nu} R_{\mu v}(\tilde{\Gamma})$, where $\tilde{\Gamma}_{\mu v}^{\lambda}$ is the connection. The matter action $S_{m}$ depends on the matter fields $\psi_{m}$ and $g_{\mu v}$. Varying Eq. (78) with respect to the metric we obtain

$$
f^{\prime}(\tilde{R}) \tilde{R}-\frac{1}{2} f(\tilde{R}) g_{\mu \nu}=0,
$$

where $^{[22]}$

$$
\tilde{R}=R+\frac{3}{2 f^{\prime}(\tilde{R})} \partial_{\lambda} f^{\prime}(\tilde{R}) \partial^{\lambda} f^{\prime}(\tilde{R})-\frac{3}{f^{\prime}(\tilde{R})} \square f^{\prime}(\tilde{R}),
$$

where $R$ is Ricci scalar constructed from the Levi-Civita connection of the metric $g_{\mu \nu}$ and the action (78) is dynamically equivalent to

$$
S=\frac{1}{2 k} \int \mathrm{d}^{4} x \sqrt{-g}\left(\Phi(R)+\frac{3}{2 \Phi} \partial_{\mu} \Phi \partial^{\mu} \Phi-V(\Phi)\right)
$$

where $\Phi=f^{\prime}(\tilde{R}), \quad V(\Phi)=\chi(\Phi) \Phi-f(\chi(\Phi))$ and $\tilde{R}=\chi(\Phi)$. This is the action of Brans-Dicke theory with the coupling parameter equal to $-3 / 2$.

In order to apply the Noether symmetry approach, one can easily verify that, in a FRW manifold, the Lagrangian related to the action (81) takes the point-like form

$$
L=12 a^{2} \phi \dot{a} \dot{\phi}+6 \phi^{2} a \dot{a}^{2}+6 a^{3} \dot{\phi}^{2}-V(\phi) a^{3}-2 k \rho_{m 0},
$$

in which we have used the redefinition $\Phi=\varphi^{2}$.

By introducing infinitesimal transformations of the time $t$, the scale factor $a$ and the function $\varphi$

$$
t^{*}=t+\varepsilon \xi_{0}(t, a, \phi), \quad a^{*}=a+\varepsilon \xi(t, a, \phi), \quad \phi^{*}=\phi+\varepsilon \eta(t, a, \phi)
$$

Noether identity on Lagrangian (82) is expressed as

$$
\frac{\partial L}{\partial t} \xi_{0}+\frac{\partial L}{\partial a} \xi+\frac{\partial L}{\partial \varphi} \eta+\frac{\partial L}{\partial \dot{a}}\left(\dot{\xi}-\dot{a} \dot{\xi}_{0}\right)+\frac{\partial L}{\partial \dot{\varphi}}\left(\dot{\eta}-\dot{\varphi}_{0}\right)+L \dot{\xi}_{0}=0 .
$$

Substituting Lagrangian (82) into Noether identity (84) leads to

$$
\begin{aligned}
& \left(24 a \phi \dot{\phi} \dot{\phi}+6 \phi^{2} \dot{a}^{2}+18 a^{2} \dot{\phi}^{2}-3 a^{2} V(\phi)\right) \xi+\left(12 a^{2} \dot{a} \dot{\phi}+12 \phi a \dot{a}^{2}-V^{\prime}(\phi) a^{3}\right) \eta \\
& +\left(12 a^{2} \phi \dot{\phi}+12 \phi^{2} a \dot{a}\right)\left(\dot{\xi}-\dot{a} \dot{\xi}_{0}\right)+\left(12 a^{2} \dot{a}+12 a^{2} \dot{\phi}\right)\left(\dot{\eta}-\dot{\phi} \dot{\xi}_{0}\right) \\
& +\left(12 a^{2} \phi \dot{a} \dot{\phi}+6 \phi^{2} a \dot{a}^{2}+6 a^{3} \dot{\phi}^{2}-V(\phi) a^{3}-2 k \rho_{m 0}\right) \dot{\xi}_{0}=0,
\end{aligned}
$$

the Eq.(85) results in

$$
\begin{gathered}
-3 a^{2} V \xi-V^{\prime} a^{3} \eta-V a^{3} \frac{\partial \xi_{0}}{\partial t}-2 k \rho_{m 0} \frac{\partial \xi_{0}}{\partial t}=0, \\
12 \varphi^{2} a \frac{\partial \xi}{\partial t}+12 a^{2} \frac{\partial \eta}{\partial t}-V a^{3} \frac{\partial \xi_{0}}{\partial a}-2 k \rho_{m 0} \frac{\partial \xi_{0}}{\partial a}=0, \\
12 a^{2} \varphi \frac{\partial \xi}{\partial t}+12 a^{2} \frac{\partial \eta}{\partial t}-V a^{3} \frac{\partial \xi_{0}}{\partial \varphi}-2 k \rho_{m 0} \frac{\partial \xi_{0}}{\partial \varphi}=0, \\
\varphi \xi+2 a \eta+2 \varphi a \frac{\partial \xi}{\partial a}-\varphi a \frac{\partial \xi_{0}}{\partial t}+2 a^{2} \frac{\partial \eta}{\partial a}=0, \\
3 \xi+2 \varphi \frac{\partial \xi}{\partial \varphi}+2 a \frac{\partial \eta}{\partial \varphi}+(a-2) \frac{\partial \xi_{0}}{\partial t}=0
\end{gathered}
$$




$$
\begin{gathered}
2 \varphi \xi+a \eta+a \varphi \frac{\partial \xi}{\partial a}+\varphi^{2} \frac{\partial \xi}{\partial \varphi}+a \frac{\partial \eta}{\partial \varphi}+a \frac{\partial \eta}{\partial a}-a \frac{\partial \xi_{0}}{\partial t}=0 \\
-12 \varphi^{2} a \frac{\partial \xi_{0}}{\partial a}+6 \varphi^{2} a \frac{\partial \xi_{0}}{\partial a}=0 \\
-12 a^{2} \frac{\partial \xi_{0}}{\partial \varphi}+6 a^{3} \frac{\partial \xi_{0}}{\partial \varphi}=0 \\
-2 \varphi^{2} a \frac{\partial \xi_{0}}{\partial \varphi}-2 a^{2} \frac{\partial \xi_{0}}{\partial a}+\varphi^{2} a \frac{\partial \xi_{0}}{\partial \varphi}=0 \\
-2 a^{2} \frac{\partial \xi_{0}}{\partial \varphi}-2 a^{2} \frac{\partial \xi_{0}}{\partial a}+a^{3} \frac{\partial \xi_{0}}{\partial a}=0 .
\end{gathered}
$$

From Eqs.(92) and (93), we have

$$
\xi_{0}=\xi_{0}(t)
$$

and then Eqs. (94) and (95) have the form $0=0$.

Substituting (96) into Eqs. (87) and (88) yields the following results:

when $a \neq \varphi$

$$
\xi=\xi(a, \phi), \quad \eta=\eta(a, \phi),
$$

and $a=\phi$

$$
\varphi \frac{\partial \xi}{\partial t}=-\frac{\partial \eta}{\partial t}
$$

This is not surprising since it has no vacuum solution. Thus, we remove the case $a \neq \varphi$ from our consideration.

The Eq.(86) results in

$$
\frac{\partial \xi_{0}}{\partial t}=-\frac{3 a^{2} V \xi+V^{\prime} a^{3} \eta}{V a^{3}+2 k \rho_{m 0}} .
$$

From Eqs.(97) and (99) one has

$$
\xi_{0}=c_{1} t+c_{2}
$$

and

$$
3 V \xi+V^{\prime} a \eta=-c_{1} V a-\frac{2 c_{1} k \rho_{m 0}}{a^{2}},
$$

i.e.

$$
a \eta=-\frac{3 V}{V^{\prime}} \xi-\frac{c_{1} V}{V^{\prime}} a-\frac{2 c_{1} k \rho_{m 0}}{V^{\prime} a^{2}} .
$$

Substituting Eqs.(100 ) and (101) into (89), we have

$$
\frac{\partial \xi}{\partial a}+\frac{\varphi}{2 a\left(\varphi-2 \frac{3 V}{V^{\prime}}\right)} \xi=\frac{a c_{1}\left(\varphi+2 \frac{V}{V^{\prime}}\right)}{2 a\left(\varphi-2 \frac{3 V}{V^{\prime}}\right)}-\frac{8 c_{1} k \rho_{m 0}}{2 V^{\prime} a^{2}\left(\varphi-2 \frac{3 V}{V^{\prime}}\right)}
$$

now, we take ${ }^{[22]} 3 V / V^{\prime}=(2 n+1) \varphi / 2 n$, i.e

$$
V=\phi^{\frac{6 n}{2 n+1}}, \quad V^{\prime}=\frac{6 n}{2 n+1} \phi^{\frac{4 n-1}{2 n+1}},
$$

and then Eq.(103) has the form

$$
\xi-\frac{1}{n} a \frac{\partial \xi}{\partial a}=\frac{5 n+1}{3 n} a c_{1}-\frac{4(2 n+1) c_{1} k \rho_{m 0}}{3 n \varphi^{\frac{6 n}{2 n+1}} a^{2}},
$$

the Eq.(105) has the following solution 


$$
\xi=f(\varphi) a^{n}+\frac{(5 n+1) c_{1}}{3(n-1)} a-\frac{4(2 n+1) c_{1} k \rho_{m 0}}{3(n+2) a^{2} \varphi^{\frac{6 n}{2 n+1}}},
$$

and

$$
\eta=-\frac{2 n+1}{2 n} \varphi f(\varphi) a^{n-1}-\frac{2 n+1}{6 n} \frac{(4 n+2) c_{1}}{(n-1)} \varphi+\frac{(2 n+1) c_{1} k \rho_{m 0}}{(n+2) a^{3} \varphi^{\frac{4 n-1}{2 n+1}}},
$$

Substituting (106) into Eq.(90), we have

$$
\frac{1}{n} \varphi \frac{d f}{d \varphi} a^{n}-\frac{n-1}{n} f(\varphi) a^{n}=-\frac{10 n^{2}-8 n-2}{3 n(n-1)} a c_{1}-\frac{56 n^{2}+32 n+2 c_{1} k \rho_{m 0}}{3(n+2) a^{2} \varphi^{\frac{6 n}{2 n+1}}}-2 c_{1}=0,
$$

Since $f(\phi)$ is the only function of variable $\phi$, then we should take constant $c_{1}=0$ into consideration. Eq.(108) is written by

$$
\frac{d f}{d \varphi}-\frac{n-1}{\varphi} f=0
$$

which has solution

$$
f(\varphi)=\varphi^{n-1},
$$

Substituting Eqs. (100), (106), (107) and (110) into (91), the infinitesimal transformations of this system are

$$
\xi_{0}=c_{2}, \quad \xi=c_{3} a^{n} \varphi^{n-1}, \quad \eta=-c_{3} \frac{2 n+1}{2 n} a^{n-1} \varphi^{n},
$$

The Lie algebras associated with the infinitesimal transformations (111) are

$$
X_{1}=\frac{\partial}{\partial t}, \quad X_{2}=a^{n} \varphi^{n-1} \frac{\partial}{\partial a}-\frac{2 n+1}{2 n} a^{n-1} \varphi^{n} \frac{\partial}{\partial \varphi} .
$$

For vector field $X_{1}$, the conserved quantity of the system (82) is:

$$
I_{1}=12 a^{2} \varphi \dot{a} \dot{\varphi}+6 \varphi^{2} a \dot{a}^{2}+6 a^{3} \dot{\varphi}^{2}-V(\varphi) a^{3}-2 k \rho_{m 0}=c_{4}=\text { const },
$$

For vector field $X_{2}$, the conserved quantity of the system (82) is:

$$
I_{2}=a^{n} \varphi^{n-} \frac{\partial L}{\partial \dot{a}}-\frac{2 n+1}{2 n} \frac{\partial L}{\partial \varphi}=-\frac{6}{n} a^{n+1} \varphi^{n} \frac{\mathrm{d}(\varphi a)}{\mathrm{d} t}=c_{5}=\text { const. }
$$

Similar to the discussions of Ref.[22], we can obtain the following solutions:

Case 1 . When $f(\tilde{R})=g(n) \tilde{R}^{3 n / n-1}$, the cosmic scale factor is

$$
\tilde{R}=g(n) a^{\frac{1-n}{n}},
$$

the time dependence of the cosmic scale factor is

$$
a(t)=\left(a_{0}+\sigma(n) t\right)^{\frac{2 n}{n-1}},
$$

where

$$
\sigma(n)=-c_{6} \frac{n^{2}}{3} \gamma(n)^{-\frac{n+1}{2}}, \gamma(n)=\frac{3 g(n) n}{n-1} G(n)^{\frac{2 n+1}{n-1}}, G(n)=\left[\frac{k \rho_{m 0}(n-1)}{g(n)(n+2)}\right]^{\frac{n-1}{3 n}} .
$$

Case 2. When $f(\tilde{R})=\alpha \tilde{R}-\lambda \alpha^{3 n / 1+2 n}$.

If $\alpha=1$, then the action is the Hilbert-Einstein action with cosmological constant $\Lambda=\lambda / 2$. It is important to note that in this case, metric and Palatini formalisms coincide. We can derive

and the conserved quantity is

$$
R=2 \lambda+\frac{k \rho_{m 0}}{a^{3}},
$$




$$
I_{3}=-\frac{6}{n}\left(\dot{a} a^{n+1}\right)=c_{6}=\text { const },
$$

so the scale factor is

$$
a=\left(a_{0}+\sigma(n)(n+2) t\right)^{\frac{1}{n+2}},
$$

in which $\sigma(n)=-n c_{6} / 6$. For $n=-2$ we have $a(t)=a_{0} e^{\delta t}$, where $\delta=c_{6} / 3$. The modified Friedmann equation in this case is $\dot{a}^{2}=\lambda a^{2} / 6+k \rho_{m 0} / 3 a$. The solution for $a(t)$ which is obtained from Noether symmetry, Eq. (121) satisfies the generalized Friedmann equation only if $n=-1 / 2, \lambda=0$ and $\sigma^{2}=3 k \rho_{m 0} / 4$. So this case is the special case of Eq. (117) when $n=-1 / 2$. Also it is easy to verify the Noether constant, which is related to gravitational constant and matter density of the universe, that is $c_{6} \sim k \rho_{m 0}$.

The conserved quantity of this system on the infinitesimal transformation (112) is

$$
I_{3}=12 a^{2} \varphi \dot{a} \dot{\varphi}+6 \varphi^{2} a \dot{a}^{2}+6 a^{3} \dot{\varphi}^{2}-V(\varphi) a^{3}-2 k \rho_{m 0}-\frac{6}{n} a^{n+1} \varphi^{n} \frac{\mathrm{d}(\varphi a)}{\mathrm{d} t}=c_{7}=\text { const } .
$$

Using conserved quantities $I_{1}$ and $I_{3}$, we can derive the cosmic scale factor $R$ and the time dependence of the cosmic scale factor $a(t)$.

\section{Conclusion}

In this paper, the Noether symmetry considering the time $t$, the scale factor $a$ and the Ricci scalar $R$ of a generic $f(R)$ cosmological model is studied. For the background geometry, the scale factor $a$ and the Ricci scalar $R$ are taken as independent dynamical variables. By employing the flat $(n+1)$-dimensional FRW metric and Lagrangian of the model in the configuration space spanned by $\{a, R\}$, the Noether's theory of $f(R)$ cosmological model has been extended into a generalized one, which contains the infinitesimal transformations with respect to the time $t$, the scale factor $a$ and the Ricci scalar $R$. On the other hand, the explicit form of the corresponding $f(R)$ function is obtained via solving the Noether identical equation. Furthermore, it is shown that this form of $f(R)$ results in a power law expansion for the scale factor. The theory of Lie symmetries of $f(R)$ cosmological models will be presented in a parallel investigation.

\section{References}

1. S. Capozziello, M. De Laurentis, S. Nojiri, and S. D. Odintsov. "Classifying and avoiding singularities in the alternative gravity dark energy models." Physical. review. D, vol.79, no.12, pp. 320-330, 2009

2. S. Nojiri and S.D. Odintsov, "Introduction to modified gravity and gravitational alternative for dark energy" Int. J. Geom. Methods Mod. Phys. Vol.4,no.1,pp.115-145, 2007

3. S. Nojiri and S. D. Odintsov. "Effective equation of state and energy conditions in phantom/tachyon inflationary cosmology perturbed by quantum effects," Physics Letters B,vol.571,no.1-2,pp. 1-10,

4. S. Nojiri and S.D. Odintsov," Unified cosmic history in modified gravity: from F(R) theory to Lorentz noninvariant models, Physics Reports, vol. 505,no.2-4,pp. 59-144, 2010

5. N.V. Dan "1/R Curvature Corrections as the Source of the Cosmological Acceleration",Phys. Rev. D,vol. 68 ,no.6, pp. 484-504, 2003 .

6. E.E. Flanagan, "Palatini Form of 1/R Gravity"Phys. Rev. Lett. vol. 92, no.7,pp. 2835-2842, 2004

7. T.P. Sotiriou, S. Liberati, "Metric-affine $f(R)$ theories of gravity," Ann. Phys. vol.322, no.4,pp.935-966,2007

8. P.J. Olver, "Applications of Lie Groups to Differential Equations,"Springer, New York, 1993.

9. L.V. Ovisiannikov, "Group Analysis of Difference Equations", Academic, New York,1982

10. N.H. Ibragimov, "Transformation Groups Applied to Mathematical Physics", Reidel, Boston, 1985.

11. G.W. Bluman, S. Kumei, "Symmetries of Differential Equations", Springer, Berlin, 1989.

12. F.X. Mei, "Applications of Lie Group and Lie Algebra to Constraint Mechanical Systems," Science Press, Beijing, 1999 (in Chinese)

13. J.L. Fu, L.Q. Chen, "Non-Noether symmetries and conserved quantities of nonconservative dynamical systems," Phys. Lett. A, vol.317 ,no. 3-4, 255-259, 2003 
14. J.L. Fu, L.Q. Chen, J. Salvador, Y.F. T ang, "Non-Noether symmetries and Lutzky conserved quantities for mechanico-electrical systems," Phys. Lett. A,vol. 358, no.1,pp. 5-10, 2006

15. J.L. Fu, L.Q. Chen and B.Y. Chen. "Noether-type theorem for discrete nonconservative dynamical systems with nonregular lattices," Sci. China: Phys. Mech. \& Astron. Vol. 53,no.3, pp.545-554, 2010

16. U. Camci and Y. Kucukakca." Noether symmetries of Bianchi I, Bianchi III, and Kantowski-Sachs spacetimes in scalar-coupled gravity theories," Phys. Rev. D, vol 76, no.76, pp. 366-367

17. A. K. Sanyal,B. Modak, C. Rubano , E. Piedipalumbo. "Noether symmetry in the higher order gravity theory,"Gen. Relativ. Gravit. Vol. 37 ,no.2, pp. :407-417, 2005

18. S. Capozziello, A. Stabile and A. Troisi. "Spherically symmetric solutions in $f(R)$ gravity via the Noether symmetry approach." Class. Quantum Grav. vol.24,no.8,pp. 2153-2166,2007

19. B. Vakili. "Noether symmetry in $\mathrm{f}(\mathrm{R})$ cosmology,"Phys. Lett. B, vol. 664,no. 1-2,pp. 16-20, 2008

20. B. Vakili. "Noether symmetric $\mathrm{f}(\mathrm{R})$ quantum cosmology and its classical correlations," Phys. Lett. B, vol.669, no.3-4,pp. 206-211, 2008

21. S. Capozziello, G. Lambiase, "Selection Rules in Minisuperspace Quantum Cosmology,"Gen. Relativ. Gravit, vol. 32,no.4,pp. 673-696,2000

22. M. Rosha and Shojai F., "Palatini [formula omitted] cosmology and Noether symmetry,"Phys. Lett. B, vol. 668 ,no.3, pp. 238-240, 2008 